\title{
Stories from COVID-19 Reveal Hospitalized Patients with Limited English Proficiency Have Always Been Uniquely Prone to Social Isolation
}

\author{
Natalie K. Kucirek, $B A^{7}$, Nicholas J. Thomas, $B A^{7}{ }^{D}$, Joshua S. Norman, $B A^{7}$, \\ Priyanka Athavale, $\mathrm{MS}^{7}$, Katrin Jaradeh, $B S^{7}$, Esther Y. Hsiang, $M D \mathrm{MBA}^{2}$, and \\ Lev Malevanchik, MD3 \\ 'University of California San Francisco School of Medicine, San Francisco, CA, USA; ${ }^{2}$ Department of Medicine, University of California San Francisco, \\ San Francisco, CA, USA; ${ }^{3}$ Division of Hospital Medicine, University of California San Francisco, San Francisco, CA, USA.
}

Through experiences with hospital visitor restrictions during the COVID-19 pandemic, a group of frontline trainees at the University of California San Francisco (UCSF) uncovered patient stories highlighting the unique challenges that patients with limited English proficiency (LEP) face in the hospital, particularly their vulnerability to social isolation. Here, we recount patient stories illustrative of this isolation, generated by insufficient professional interpreter use, ad hoc interpretation, and scarcity of media in preferred languages. When confronted with the social isolation faced by all patients during COVID-19, we more clearly saw the healthcare disparities affecting patients with LEP. A trainee-led videoconferencing initiative facilitating social calls between patients at UCSF and their loved ones proved especially helpful in reducing the disconnection that patients with LEP experience in the hospital. Motivated by the findings of this project, we advocate for other institutions to take similar action, such as hiring inpatient telehealth navigators and providing tablets for ad lib use. Enacting these changes will keep patients with LEP connected to their families and communities while in the hospital, an essential step towards establishing an equitable experience for patients with LEP.

KEY WORDS: limited English proficiency; COVID-19; telehealth; health equity; interpreter use.

J Gen Intern Med 36(3):786-9

DOI: $10.1007 / \mathrm{s} 11606-020-06383-\mathrm{Z}$

(C) Society of General Internal Medicine 2021

$\mathrm{T}$ he COVID-19 pandemic has had a profound impact on patient experience in the inpatient setting. Early in the pandemic, health systems implemented strict visitation restrictions to mitigate the spread of SARS-CoV2. ${ }^{1}$ These policies disrupted the usual patient experience in the hospital, changing

Prior Presentations None.

Received August 16, 2020

Accepted December 1, 2020

Published online January 6, 2021 the ways in which patients received bad news, made decisions, and involved family in their care. ${ }^{2}$ This led to highly visible, tragic moments of isolation for patients, especially at the end-oflife and in critical care settings. In response, residents and medical students at the University of California San Francisco (UCSF) developed and implemented Connecting During COVID, a project that facilitated videoconference visits between patients and their loved ones on donated tablets at three hospital sites. Over the course of our initiative at the UCSF Parnassus hospital, we observed that nearly $30 \%$ of patients utilizing our service had limited English proficiency (LEP), despite comprising only 20\% of the 600-bed hospital population. This exposed an unmet need for patients with LEP, namely assistance maintaining social connections while in the hospital.

Nationally, $20 \%$ of the US population speaks a language other than English at home and $8.6 \%$ of individuals in the USA are classified as having LEP, thus comprising a substantial portion of hospital patient populations. ${ }^{3}$ While professional interpretation services are widely available for medical teams and staff to convey clinical updates, they remain underutilized. ${ }^{4,5}$ Consequently, patients with LEP face barriers communicating their medical needs to nurses and physicians in the hospital setting. This leaves patients with LEP with a poorer understanding of medical encounters. ${ }^{6}$ Furthermore, we conjecture that these patients have limited non-clinical social interactions with hospital staff and volunteers, who are often primarily English-speaking. They may also have fewer enriching experiences through provided digital and print media, typically only available in a limited number of languages. Our personal narratives of connecting patients with loved ones through the Connecting During COVID project highlight the importance of reducing social isolation and enriching the inpatient experience for limited English speakers. This is of particular importance as epidemiologic data show that racial and ethnic minorities in the USA have been disproportionately affected by the pandemic. . $^{7-9}$

\section{CONNECTING DURING COVID: PROJECT OVERVIEW}

Through the generous donation of tablets from the community, our team of frontline trainees launched a consult service in 
which providers sent requests to medical students, furloughed from clinical duties due to the pandemic, to coordinate video calls with families. Through our intervention at the UCSF Medical Center, we facilitated 150 calls over a 60 -day period. We assisted 70 individual patients, 30\% ( $n=21)$ of whom were identified as non-English-speaking by the electronic medical record. We reached patients who spoke English, Spanish, Mandarin, Cantonese, Greek, Tagalog, Samoan, and Farsi with an average age of 70 years old.

\section{SOCIAL ISOLATION AND PATIENTS WITH LIMITED ENGLISH PROFICIENCY}

The following are vignettes written by medical students implementing Connecting During COVID that characterize the inpatient experience for patients with LEP during the pandemic. For anonymity, names and exact ages have been changed.

A care team member flagged me down in the hallway and told me about a 70-year-old Tagalog-speaking patient who was very lonely. The provider was having trouble expressing to the patient why she couldn't see her family due to the pandemic and was hoping her loved ones could help explain what was going on. The family did not speak English and the care team member, overwhelmed with clinical responsibilities, was unable to orchestrate a call with an interpreter over the phone.

Due to our evolving understanding of COVID-19 transmission compounded by language barriers, it was difficult to describe the rationale behind COVID-19 visitation restrictions. Consequently, patients with LEP were often confused as to why family members were unable to visit, leading to further isolation and distress. Our shortcomings conveying COVID-19-related changes to the inpatient experience point to preexisting challenges with under-communication of nuanced and complex issues common in hospital care. Patients with LEP will continue to face these issues beyond the pandemic if not carefully addressed.

I was connecting a 60-year-old, Spanish-speaking patient who suffered a stroke in the intensive care unit (ICU) that paralyzed his arms. After muting the English-speaking sports commentators on his television, I introduced myself and was met with a vacant stare. His nurse said, "He isn't verbal really, but now would be a fine time to make a call if the family wants to see him." Once his family was on video, I approached the patient's bed and held the tablet in front of his face. Immediately the energy in the room changed - the formalities of introductions and full titles of the hospital fell away to familiarities and nicknames. He brightened and was even occasionally responsive with one-word answers. The nurse remarked to the family how much he perked up when they got on video, adding that he must enjoy hearing their voices and seeing their faces. The family asked her if she uses an interpreter to speak to him. The nurse replied honestly, "I do whenever we talk as a team, but not throughout the day." The wife asked, "Would you mind using the interpreter whenever you speak to him? He only understands some English and may have trouble understanding after the stroke."

Research has shown that isolation increases hospital readmissions and can predict functional decline and death in older persons. ${ }^{10,11}$ This isolation and lack of stimulation can also contribute to delirium, especially in an ICU, and can lead to poor patient outcomes. ${ }^{12-14}$ In the COVID-19 era of visitor restrictions, we observed an exacerbation of these vulnerabilities in patients with LEP, especially those who were older and lacked access to personal technology and in-hospital media in their native language. Overwhelming clinical duties during a shift may contribute to the care team using professional interpretation only when necessary, leading to fewer social interactions and opportunities for patients with LEP to convey needs.

I was called to connect Ms. C, a Spanish-speaking patient in her 90s, with her daughter. The pair quickly started chatting once I initiated the video call, eager to reconnect after being physically distant. Although her nurse and I spoke Spanish, Ms. C's daughter expanded upon her mother's desires, instructing us to adjust the bed to maximize her comfort. When I returned an hour later, they were still talking animatedly. A physical therapist entered and I offered to end the call so that she could start her session with Ms. C. The physical therapist suggested keeping her daughter on video during the sessions to encourage her mother. When I returned, the physical therapy session had been completed successfully with the daughter's virtual presence - the therapist was struck by how smoothly the session went compared to prior sessions.

Even with language-concordant providers and formal interpretation, loved ones still valued the opportunity to express their family members' concerns to the team. Observing English-speaking family members advocating for patients with LEP during our video calls underscored the importance of the support networks these patients maintain outside of the hospital. The tablets provided a means by which these support systems could be brought into the hospital. We discourage using family for ad hoc interpretation but acknowledge that loved ones have valuable insights about a patient's needs and cultural context that can improve their care when communicated to the medical team. 


\section{LIMITATIONS TO HELPING PATIENTS WITH LEP CONNECT STILL EXIST}

Despite the success stories we experienced through the Connecting During COVID project, several barriers persisted in connecting patients with family and friends.

I hoped to teach Mr. Y, the family member of a Mandarin-speaking patient, how to use our videoconferencing app to schedule a call with his loved one who was hospitalized. After twenty minutes speaking to me with an interpreter, Mr. Y became frustrated and asked a separate bilingual friend to coordinate with me in English. This friend explained Mr. Y did not know how to use a computer well enough to video call his hospitalized loved one. Also, Mr. Y still had to physically go to work during COVID-19 and lacked access to informal familial tech support, unlike many other patients who had technologically savvy children using videoconferencing for schoolwork. In fact, he would likely not have a scheduled break from work to take a call.

Mr. Y's experience underscores that while technology is a valuable tool for reducing isolation in the hospital, factors such as limited English proficiency and unfamiliarity with novel technology may prevent some users from reaping its benefits. Mr. Y's story also more broadly highlights the importance of community support for our patients and families with LEP in navigating systems often designed primarily with English-speaking users in mind. When these support systems are physically distant from the bedside, either due to COVID-19 or because busy work schedules or long travel distances to hospitals prevent frequent visitation, patients with LEP face further isolation.

Although some preexisting services exist in the hospital for videoconferencing, patients and families continuously relied on our team to stay connected to their families. This indicates that providing access to technology without assistance navigating its complexities may be insufficient for patients. Moreover, many hospitalized patients and family members with LEP are older and face not only a language barrier but also a technological barrier in using video-based applications. Telehealth-based solutions to social isolation in the hospital must address these overlapping challenges for patients with LEP to mitigate the gaps in connectivity during their inpatient stay. A recent study found that patients with a non-English language preference were significantly less likely to use telemedicine compared with English speakers. ${ }^{15}$ Although our initiative used videoconferencing for social rather than clinical purposes, we must take care to present new technology in an accessible way in any medical setting to avoid exacerbating healthcare inequities.

\section{CALL TO ACTION, LESSONS LEARNED, AND CONCLUSION}

The COVID-19 pandemic forced social isolation upon all our patients and consequently challenged us to think deeply about the critical need for social connection to enhance the patient care experience. Patients at urban academic medical centers are already at increased risk for isolation, as many patients and families travel great distances to access quaternary care. However, patients with LEP face an additional, unique set of challenges in the inpatient setting that worsen social isolation. Such factors include under-interpretation of complex conversations, non-universal use of interpreters, fewer conversations throughout the day with staff, and a paucity of media in nonEnglish languages. Furthermore, hospitalization often separates patients with LEP from the social support networks particularly important in navigating systems designed with English speakers in mind. We assert that increasing the routine use of professional interpreters during clinical care is not enough. Patients with LEP deserve an equitable hospital experience in which all clinical conversations are translated into their preferred language and they have opportunities to connect socially throughout their stay.

Therefore, we must think creatively to develop interventions to help patients with LEP stay connected to their loved ones. One solution that is currently being piloted at UCSF is a dedicated "Inpatient Video Navigator." Providers can consult the navigator to teach families and patients how to set up telehealth meetings with clinicians and social video visits while the patient is in the hospital. Moreover, a tablet available for patients to use at-will could facilitate engagement with media (online newspapers, entertainment, TV stations with current events) in a patient's native language. Hospitals must take care, however, to ensure that such interventions are not a substitute for medical interpretation, and that loved ones brought virtually to the bedside do not serve as ad hoc interpreters.

As videoconferencing and telehealth become more prevalent in patient care, we must thoughtfully prioritize connecting the most difficult to reach LEP, elderly, and technologically challenged patients to avoid worsening preexisting health disparities. Our initiative highlights that careful implementation of tablet technology for social connection helps to address barriers to communication that patients with LEP face. Although this intervention is one model, we must seek further solutions to ensure parity of experience in our hospitals, regardless of the languages our patients speak.

Acknowledgments: The authors wish to thank all the medical students, residents, and faculty advisors who have spent tremendous hours working on the Connecting During COVID Project. Additionally, the authors thank Margaret Handley $\mathrm{PhD} M P H$, and Smitha Ganeshan MD MBA for help with editing this article.

Corresponding Author: Nicholas J. Thomas, BA; University of California San Francisco School of Medicine, San Francisco, CA, USA (e-mail: nicholas.thomas@ucsf.edu). 
Funding This work led to a grant from the Flora Family Foundation.

\section{Compliance with Ethical Standards:}

Conflict of Interest: The authors declare that they do not have a conflict of interest.

Ethical Approval: Not applicable.

\section{REFERENCES}

1. Lewnard JA, Lo NC. Scientific and ethical basis for social-distancing interventions against COVID-19. Lancet Infect Dis. 2020;20(6):631-633. https://doi.org/10.1016/S1473-3099(20)30190-0

2. Hafner K. 'A Heart-Wrenching Thing': Hospital Bans on Visits Devastate Families. The New York Times. https://www.nytimes.com/2020/ 03/29/health/coronavirus-hospital-visit-ban.html. Published March 29, 2020. .

3. Betancourt JR, Renfrew MR, Green AR, et al. Improving patient safety systems for patients with limited English proficiency: a guide for hospitals. (Prepared by the Disparities Solutions Center, Mongan Institute for Health Policy at Massachusetts General Hospital and Abt Associates, Cambridge, MA, under Contract No. HHSA290200600011I) Rockville, MD: Agency for Healthcare Research and Quality; July 2012. AHRQ Publication No. 12-0041. September 2012.

4. Schenker Y, Pérez-Stable EJ, Nickleach D, Karliner LS. Patterns of interpreter use for hospitalized patients with limited English proficiency. $J$ Gen Intern Med. 2011;26(7):712-717. https://doi.org/10.1007/ s11606-010-1619-Z

5. López L, Rodriguez F, Huerta D, Soukup J, Hicks L. Use of interpreters by physicians for hospitalized limited English proficient patients and its impact on patient outcomes. J Gen Intern Med. 2015;30(6):783-789. https://doi.org/10.1007/s11606-015-3213-x

6. Wilson E, Chen AHM, Grumbach $\mathbf{K}$, Wang F, Fernandez A. Effects of limited English proficiency and physician language on health care comprehension. J Gen Intern Med. 2005;20(9):800-806. https://doi. org/10.1111/j.1525-1497.2005.0174.x
7. Alcendor DJ. Racial disparities-associated COVID-19 mortality among minority populations in the US. Journal of Clinical Medicine. 2020;9(8):2442. https://doi.org/10.3390/jcm9082442

8. Chowkwanyun M, Reed AL. Racial Health Disparities and Covid-19Caution and Context. New England Journal of Medicine. 2020;383(3):201-203. https://doi.org/10.1056/NEJMp2012910

9. Tai DBG, Shah A, Doubeni CA, Sia IG, Wieland ML. The Disproportionate Impact of COVID-19 on Racial and Ethnic Minorities in the United States. Clin Infect Dis.https://doi.org/10.1093/cid/ciaa815

10. Newall N, McArthur J, Menec VH. A Longitudinal Examination of Social Participation, Loneliness, and Use of Physician and Hospital Services: Journal of Aging and Health. Published online October 5, 2014. https:// doi.org/10.1177/0898264314552420

11. Perissinotto CM, Stijacic Cenzer I, Covinsky KE. Loneliness in older persons: a predictor of functional decline and death. Arch Intern Med 2012;172(14):1078-1083. https://doi.org/10.1001/archinternmed. 2012.1993

12. Kotfis K, Williams Roberson S, Wilson JE, Dabrowski W, Pun BT, Ely EW. COVID-19: ICU delirium management during SARS-CoV-2 pandemic. Critical Care. 2020;24(1):176. https://doi.org/10.1186/s13054-02002882-x

13. McCusker J, Cole M, Abrahamowicz M, Han L, Podoba JE, RammanHaddad L. Environmental Risk Factors for Delirium in Hospitalized Older People. Journal of the American Geriatrics Society. 2001;49(10):13271334. https://doi.org/10.1046/j.1532-5415.2001.49260.x

14. Pompei P, Foreman M, Rudberg MA, Inouye SK, Braund V, Cassel CK Delirium in hospitalized older persons: outcomes and predictors. $J$ Am Geriatr Soc. 1994;42(8):809-815. https://doi.org/10.1111/j.1532-5415. 1994.tb06551.x

15. Reed ME, Huang J, Graetz I, et al. Patient Characteristics Associated With Choosing a Telemedicine Visit vs Office Visit With the Same Primary Care Clinicians. JAMA Netw Open. 2020;3(6):e205873-e205873. https:// doi.org/10.1001/jamanetworkopen.2020.5873

Publisher's Note: Springer Nature remains neutral with regard to jurisdictional claims in published maps and institutional affiliations. 